\title{
RECIDIVA DEL CÁNCER DE PRÓSTATA DESPUÉS DE LA PROSTATECTOMÍA RADICAL Y RADIOTERAPIA DE RESCATE
}

\author{
A. OJEA CALVO, A. PÉREZ RODRÍGUEZ, F. DOMÍNGUEZ FREIRE, \\ A. ALONSO RODRIGO, B. RODRÍGUEZ IGLESIAS, J. BENAVENTE DELGADO, \\ J.M. BARROS RODRÍGUEZ, A. GONZÁLEZ PIÑEIRO, M. OTERO GARCÍA, \\ V. MUÑOZ GARZÓN
}

Complejo Hospitalario Universitario Xeral-Cies de Vigo. Pontevedra.

Actas Urol Esp. 28 (10): 743-748, 2004

\section{RESUMEN}

RECIDIVA DEL CÁNCER DE PRÓSTATA DESPUÉS DE LA PROSTATECTOMÍA RADICAL Y RADIOTERAPIA DE RESCATE

OBJETIVO: Evaluar la eficacia de la radioterapia en el lecho prostático en pacientes con cáncer de próstata y fracaso bioquímico después de la prostatectomía radical.

MATERIAL Y MÉTODOS: Analizamos los resultados de 292 pacientes a los que se le practicó prostatectomía radical por cáncer de próstata localizado T1-T2, entre enero de 1992 y junio de 2003, con un seguimiento medio de 36 meses (rango 6 meses a 12 años). Se detecta fracaso bioquímico (PSA > 0,20 ng/ml) en 75 (26\%) pacientes. De los 75 pacientes con fracaso bioquímico, 9 (12\%) se diagnosticó de recidiva local siguiendo los siguientes criterios: a) Primer PSA obtenido a las 6 semanas de la intervención $<0,20 \mathrm{ng} / \mathrm{ml}$. b) Tiempo hasta el fracaso bioquímico $>6$ meses. c) Tiempo de duplicación del PSA $>6$ meses. d) Velocidad de PSA después de la prostatectomía radical $<0,75 / \mathrm{ng} / \mathrm{ml} / \mathrm{año}$. e) Nivel de PSA después de la prostatectomía radical $<2,5 \mathrm{ng} / \mathrm{ml}$. Los 9 pacientes diagnosticados de recidiva local reciben una dosis media de 56,42 Gy en el lecho prostático.

RESULTADOS: De los 9 pacientes diagnosticados de recidiva local, en $7(77,7 \%)$ se obtuvo una respuesta completa durante un tiempo medio de seguimiento de 25 meses (6-30 meses). El tiempo entre la radioterapia y la respuesta, en los pacientes con respuesta completa, siempre fue inferior a los 3 meses. No se observaron efectos adversos importantes secundarios a la radioterapia.

CONCLUSIONES: La radioterapia de rescate puede ser beneficiosa en un seleccionado grupo de pacientes con recidiva local. La cinética del PSA después de la prostatectomía radical es útil para distinguir las recidivas locales de las metástasis a distancia.

PALABRAS CLAVE: Prostatectomía radical. Recidiva local. Radioterapia de rescate.

\section{ABSTRACT}

PROSTATE CANCER RECURRENCE AFTER RADICAL PROSTATECTOMY AND SALVAGE RADIOTHERAPY

OBJETIVE: To evaluate the efficacy of the radiotherapy to prostatic bed in patients with biochemical recurrence for prostate cancer after radical prostatectomy.

MATERIAL AND METHODS: We analyse the results of 292 patients underwent radical prostatectomy for localized prostate cancer T1-T2 between January 1992 and June 2003, with an average folow-up of 36 months (range 6 months to 12 years). We detect biochemical recurrence (PSA $>0.20 \mathrm{ng} / \mathrm{ml}$ ) in $75(26 \%)$ patients. Of 75 patients with biochemical recurrence, 9 (12\%) was diagnosed of local recurrence by the following criteria: a) The first PSA obtained 6 weeks after radical prostatectomy $<0.20 \mathrm{ng} / \mathrm{ml}$. b) The time to biochemical recurrence $>6$ months. c) The prostate specific antigen doubling time $>6$ months. d) The prostate specific antigen velocity after radical prostatectomy $<0.75$ $\mathrm{ng} / \mathrm{ml} /$ year. e) The prostate specific antigen level after radical prostatectomy $<2.5 \mathrm{ng} / \mathrm{ml}$. The 9 patients diagnosed of local recurrence received an average dose of 56.42 Gy in the prostate bed.

RESULTS: Of all 9 patients with local recurrence, $7(77.7 \%)$ has complete response with an average time of followup of 25 months (6-30 months). The time between the radiotherapy and the response, in patients with complete response, was lower than 3 months. Were not observed significant adverse effects associated to radiotherapy.

CONCLUSIONS: The salvage radiotherapy may be beneficial in select patients with local recurrence. The characteristics of prostate specific antigen elevation are useful in distinguishing men with local recurrence from those with distant metastases.

KEY WORDS: Radical prostatectomy. Local recurrence. Salvage radiotherapy. 
$\mathrm{L}$ a recidiva del cáncer de próstata después de la prostatectomía radical se sitúa en torno al $30 \%$ de los pacientes. Aunque se han descrito casos a los 10 y 15 años de la intervención, suele manifestarse entre los 19 y 24 meses de la intervención quirúrgica. Se diagnostica por la elevación del PSA por encima de $0,2 \mathrm{ng} / \mathrm{ml}$, que es lo que se denomina con el termino de fracaso bioquímico o fracaso del PSA. El análisis de la cinética del PSA o comportamiento del PSA postoperatorio es el mejor método para determinar la localización de la recidiva, clasificándola en local o metastásica $^{1-7}$. Un tiempo hasta el fracaso del $P S A$ superior a 6 meses predice recidiva local y menor de 6 meses predice diseminación metastásica. Una velocidad del PSA elevándose después de la cirugía a un ritmo de menos de 0,75 $\mathrm{ng} / \mathrm{ml} / \mathrm{año}$ es muy significativa de recidiva local mientras que si se eleva a una velocidad de más de $0,75 \mathrm{ng} / \mathrm{ml} /$ año es sinónimo de diseminación metastásica. Un tiempo de duplicación del PSA superior a 6 meses indica recidiva local y el inferior a 6 meses recidiva sistémica. La cantidad de PSA también es útil para hacer el diagnóstico entre recidiva local y sistémica. PSA inferior a 2,5 $\mathrm{ng} / \mathrm{ml}$ es significativo de recidiva local y superior a $2,5 \mathrm{ng} / \mathrm{ml}$ de recidiva sistémica ${ }^{1-11}$. Las técnicas por imagen disponibles actualmente, gammagrafía ósea, tomografía axial computarizada, resonancia magnética, radioinmunocentellografía, tomografía por emisión de positrones, demuestran poca utilidad en el diagnóstico precoz de la recidiva y en la localización de la recidiva ${ }^{4}$. La biopsia del lecho prostático es poco eficaz para localizar la recidiva en pacientes con PSA inferior a $1 \mathrm{ng} / \mathrm{ml}$. El tratamiento con intención curativa de la recidiva local es la radioterapia del lecho prostático $^{4}$.

El objetivo de este estudio es valorar la capacidad de la radioterapia de rescate para erradicar la recidiva local diagnosticada por la cinética del PSA.

\section{MATERIAL Y MÉTODOS}

Se analiza la evolución de 292 pacientes con cáncer de próstata T1-T2 a los que se le practicó prostatectomía radical retropúbica entre enero de 1992 y junio de 2003. El tiempo de seguimiento medio es de 36 meses (6 meses-12 años).
Los tumores se reclasificaron según el sistema TNM de 2002 sexta edición de la UICC ${ }^{12}$. En todos los pacientes se establecieron controles periódicos con determinación de PSA en sangre a las 6 semanas de la intervención y posteriormente cada 6 meses de forma indefinida. En caso de PSA superior a $0,20 \mathrm{ng} / \mathrm{ml}$ en al menos dos determinaciones se diagnosticaba al paciente de fracaso bioquímico. El PSA se determinó con el ensayo KRYP$\mathrm{TOR}^{\mathrm{R}}$ que se fundamenta en la tecnologia TRACE ${ }^{\mathrm{R}}$ (Time Resolved Amplified Cryptate Emission).

Se detectó fracaso bioquímico en 75 pacientes lo que representa un porcentaje del 26\%. Se observó fracaso bioquímico en el 10\% (2/20) de los tumores pTO, en el 11\% (8/74) de los tumores pT2a, en el 12\% (8/67) de los tumores pT2b, en el $21 \%(7 / 34)$ de los tumores pT2c, en el $28 \%$ (19/61) de los tumores pT3a, en el 74\% (14/19) de los tumores pT3b, en el 100\% (2/2) de los tumores pT4 y en el 100\% (15/15) de los tumores pT2-3N 1 .

Para hacer el diagnóstico de recidiva local del cancer de próstata por la cinética del PSA postoperatorio se seleccionó a los pacientes que cumplían todos los criterios siguientes:

1. Que el PSA hubiese descendido a cifras insignificantes a las 6 semanas de la prostatectomía (PSA postoperatorio $<0,20 \mathrm{ng} / \mathrm{ml}$ ).

2. Que el tiempo hasta el fracaso bioquímico (PSA > de 0,20ng/ml) fuese mayor de 6 meses.

3. Que el tiempo de duplicación del PSA fuese mayor de 6 meses.

4. Que la velocidad del PSA fuese inferior a $0,75 \mathrm{ng} / \mathrm{ml} / \mathrm{año}$.

5. Que el PSA no sobrepasase la cifra de 2,5 $\mathrm{ng} / \mathrm{ml}$.

El número de pacientes con recidiva local diagnosticada por la cinética del PSA fue de 9, lo que representa el $12 \%$ de todos los pacientes con fracaso bioquímico: $1(11,1 \%)$ se correspondía con un tumor pTa, $2(22,2 \%)$ con tumor pT2b, 2 $(22,2 \%)$ con tumor pT2c, $2(22,2 \%)$ con tumor pT3a, $1(11,1 \%)$ con tumor pT3b y $1(11,1 \%)$ con tumor pT4a. La edad media de los pacientes con recidiva local era de 58 años que se siguieron durante un tiempo medio de 50 meses (18-106). La mediana de tiempo hasta la progresión biológica era de 17 meses (12-44meses). La mediana 
de tiempo de duplicación del PSA era de 12 meses (12-48 meses). La mediana de la velocidad de PSA en la recidiva bioquímica era de $0,25 \mathrm{ng} / \mathrm{ml} / \mathrm{año}$ $(0,05-0,74)$. La mediana de PSA previo a la radioterapia era de $1,04(0,37-2,40)$ (Tabla 1$)$.

Para el tratamiento de radioterapia de rescate se utilizaron fotones de $18 \mathrm{MV}$ de acelerador lineal. El volumen blanco incluía el lecho de prostatectomía, utilizando 4 campos en caja conformados con multiláminas isocéntricas. Se administró una dosis media de radioterapia de 56,42 Gy en 5 fracciones semanales de 2 Gy.

Se determina el porcentaje de respuestas completas hasta cifras de PSA no significativas (PSA< $0,20)$ y el tiempo que transcurre desde que se administra la radioterapia y se alcanza la respuesta completa. También se calcula la duración de la respuesta completa. Para el manejo de datos y el análisis de los resultados empleamos el programa informático, SPSS para Windows, de Microsoft.

\section{RESULTADOS}

El número de pacientes con respuesta completa a la radioterapia local fue de $7(77,8 \%)$. El tiempo que transcurrió desde que se administró la radioterapia hasta que se alcanzaron cifras de $\mathrm{PSA}<$ de $0,20 \mathrm{ng} / \mathrm{ml}$ fue inferior a 3 meses en todos los pacientes que respondieron. Los pacientes que respondieron lo hicieron durante una mediana de tiempo de 25 meses (6-30 meses) y no se observaron nuevas recaídas en los pacientes que respondieron. Los efectos secundarios de la radioterapia se manifestaron con sintomatología intestinal, rectal o vesical leve en 5 (56\%) de los pacientes que fueron controlados con tratamiento sintomático (Tabla 1).

\section{COMENTARIOS}

El tratamiento de la recidiva local del cáncer de próstata después de la prostatectomía radical es la radioterapia del lecho prostático con intención curativa ${ }^{13}$. Hasta la actualidad los métodos más frecuentemente utilizados con la pretensión de localizar la recidiva tumoral han sido: el análisis del comportamiento del PSA después de la intervención o cinética del PSA postoperatorio, la gammagrafía ósea, la tomografía axial computarizada, la resonancia magnética, la radioinmunocentellografía, la tomografía por emisión de positrones y la biopsia del lecho prostático.

\section{Cinética del PSA postoperatorio}

La determinación en sangre del PSA es el método más simple y específico para detectar la presencia de tumor residual después de la prostatectomía radical. El primer control se suele realizar a las 6 semanas de la intervención, para evitar

Tabla 1

\begin{tabular}{ccccccccc}
\hline Paciente & $\begin{array}{c}\text { Categoría } \\
\text { PT }\end{array}$ & Edad & Gleason & $\begin{array}{c}\text { PSA previo a la } \\
\text { a la radioterapia } \\
\text { (ng/ ml) }\end{array}$ & $\begin{array}{c}\text { Tiempo de } \\
\text { duplicación } \\
\text { PSA meses }\end{array}$ & $\begin{array}{c}\text { PSA } \\
\text { velocidad } \\
\text { (ng/ ml/ año) }\end{array}$ & $\begin{array}{c}\text { Respuesta } \\
\text { completa a la } \\
\text { radioterapia }\end{array}$ & $\begin{array}{c}\text { Tiempo de } \\
\text { seguimiento } \\
\text { con respuesta } \\
\text { a la radioterapia } \\
\text { (meses) }\end{array}$ \\
\hline 1 & pT3a & 48 & 6 & 1,71 & 36,00 & 0,16 & No & No-respuesta \\
2 & pT4a & 62 & 9 & 0,76 & 24,00 & 0,25 & Sí & 30,00 \\
3 & pT2c & 59 & 7 & 0,37 & 48,00 & 0,12 & Sí & 12,00 \\
4 & pT2a & 62 & 6 & 1,48 & 24,00 & 0,74 & Sí & 25,00 \\
5 & pT2b & 54 & 6 & 1,05 & 12,00 & 0,20 & Sí & 30,00 \\
6 & pT2c & 59 & 6 & 0,89 & 12,00 & 0,25 & Sí & 27,00 \\
7 & pT3a & 52 & 6 & 1,04 & 12,00 & 0,27 & Sí & 24,00 \\
8 & pT2b & 63 & 8 & 0,62 & 12,00 & 0,05 & Sí & 6,00 \\
\hline
\end{tabular}

Características de los 9 pacientes que recibieron radioterapia de rescate después del fracaso bioquímico y resultados. El nivel de PSA previo a la radioterapia era de 1,04 (0,37-2,40); el tiempo de duplicación del PSA de 12 meses (12-48); la velocidad del PSA de 0,25 ng/ml(0,05-0,75). El número de pacientes rescatados fue de 7 ( $77,8 \%)$ con un tiempo de seguimiento de 25 meses (6-30 meses). 
falsos positivos. Por que hay que tener en cuenta que la vida media del PSA es de 2,6 días y que durante la intervención quirúrgica existe la posibilidad que una gran cantidad de PSA se libere y pase a la sangre. Si el tumor está localmente avanzado o el Gleason es elevado los controles se continúan semestralmente los 2 ó 3 primeros años y posteriormente cada año de forma indefinida. Si el tumor está localizado y el Gleason es intermedio o bajo, los controles de PSA pueden practicarse anualmente de forma indefini$\mathrm{da}^{4,14,15}$. Aunque no existe un consenso que defina cual es el nivel de PSA insignificante, la idea más generalizada es que una cifra de PSA de 0,1 o inferior, con los ensayos convencionales, se debe a un artefacto que puede provenir de las glándulas periuretrales. Niveles de PSA de 0,2 $\mathrm{ng} / \mathrm{ml}$ o superiores son patológicos e indican presencia de tumor residual. Niveles de PSA entre 0,1 y $0,2 \mathrm{ng} / \mathrm{ml}$ son cifras que están en terreno de nadie, que sólo se consideran patológicos a posteriori, cuando el PSA sobrepasa los 0,20 $\mathrm{ng} / \mathrm{ml}$ en los controles posteriores. Entonces la cifra de PSA entre 0,1 y 0,20 ng/ml se aprovecha para estimar el tiempo de duplicación de $\mathrm{PSA}^{4}$.

El análisis de la cinética del PSA postoperatorio se consideró una buena forma de localizar el lugar de la recidiva. Partin AW et al. en $1994^{9}$ analizaron la utilidad para localizar el lugar de la recidiva del tiempo que transcurría desde la prostatectomía hasta el fracaso del PSA y de la velocidad de ascenso del PSA una vez que se había producido el fracaso bioquímico. De una serie de 1.058 prostatectomías radicales los autores siguieron a 51 pacientes con fracaso bioquímico, hasta la aparición de recidiva local o la aparición de metástasis. De los 51 pacientes, 16 (31\%) recidivaron localmente y 35 (69\%) lo hicieron con metástasis a distancia. El intervalo de tiempo desde la prostatectomía radical hasta el fracaso del PSA fue más largo en los pacientes con recidiva local que en los que tenían metástasis. Ningún paciente con recidiva local registró un PSA detectable dentro de los 6 meses siguientes a la cirugía frente al 50\% de los que desarrollaron metástasis a distancia. En el mismo trabajo, también se observó que la velocidad de ascenso del PSA era distinta en los pacientes con recidiva local y en los pacientes con metástasis. En el 94\% de los pacientes con recidiva local el PSA crecía a un ritmo inferior a $0,75 \mathrm{ng} / \mathrm{ml} /$ año frente al $46 \%$ de los pacientes que presentaban metástasis a distancia. El tiempo de duplicación del PSA fue analizado por diversos autores, con intención de localizar la recidiva tumoral. Patel A et al. ${ }^{10}$ analizaron 77 pacientes con fracaso de PSA después de la prostatectomía radical. En 33 pacientes el tiempo de duplicación del PSA era inferior a 6 meses y en 44 era superior a 6 meses. En 11 (33\%) de los pacientes con tiempo de duplicación de PSA inferior a 6 meses observaron metástasis a distancia frente a 1 (3\%) que presentaba recidiva local. Cuando el tiempo de duplicación del PSA era superior a los 6 meses solamente en 1 (2\%) de los pacientes se observó metástasis a distancia frente a 8 (18\%) que presentaban recidiva local. Pound CR et al. ${ }^{16}$ observaron que un tiempo de duplicación del PSA inferior a los 10 meses era la variable que mejor predecía la aparición de enfermedad metastásica. Roberts SG et al. ${ }^{17}$ concluyen en un análisis univariante y multivariante que el tiempo de duplicación del PSA era la variable con más capacidad para discernir entre la recidiva local y la enfermedad metastásica después de la prostatectomía radical. El valor del nivel de PSA en sangre para predecir el lugar de la recidiva fue estudiado por Wu JJ et al. en $1995^{11}$. Analizaron los resultados de la radioterapia local en un grupo de 53 pacientes con fracaso bioquímico, 27 con niveles de PSA inferiores a $2,5 \mathrm{ng} / \mathrm{ml}$ y 26 con niveles de PSA superiores a $2,5 \mathrm{ng} / \mathrm{ml}$. Vieron que 14 (52\%) de los pacientes con PSA menor de 2,5 respondían a la radioterapia mientras que sólo respondían 2 (8\%) de los pacientes con niveles de PSA superiores a $2,5 \mathrm{ng} / \mathrm{ml}$.

\section{Técnicas por imagen}

Diferentes técnicas por imagen han sido evaluadas para detectar y localizar la recidiva después de la prostatectomía radical. La gammagrafía ósea fue estudiada por Cher ML et al. en $1998^{18}$ en una serie de 93 pacientes. La posibilidad de que la gammagrafía ósea fuese positiva era inferior al $5 \%$ en pacientes con PSA por debajo de $40 \mathrm{ng} / \mathrm{ml}$. La tomografía axial computarizada fue utilizada por Older RA et al. en $1995^{19}$ comparando las imágenes del lecho quirúrgico de 11 pacientes con fracaso del PSA con otros pacientes sin 
fracaso del PSA que actuaban como control. No observaron diferencias significativas en las imágenes de los lechos quirúrgicos de los 2 grupos. Kramer S et al. en $1997^{20}$ observaron que la tomografía axial computarizada solamente diagnosticaba el 36\% de las recidivas locales confirmadas con biopsia. La tomografía axial computarizada sólo podía reconocer las recidivas locales de más de 2 $\mathrm{cm}$. La radioinmunocentellografía fue evaluada por Kahn D et al. en $1998^{21}$. Comunicaron los resultados del análisis de 183 pacientes y la radioinmunocentellografía detectó recidiva en la celda prostática sólo en el 35\% de los casos en los que ya estaba confirmada la recidiva local por biopsia. Utilizando la biopsia como patrón oro, la sensibilidad de la radioinmunocentellografía era del $49 \%$ y la especificidad del $77 \%$, con un valor predictivo positivo del 50\% y negativo del 70\%. Raj GV et al. en $2002^{22}$ concluyeron en su análisis que la radioinmunocentellografia era superior a la tomografia axial computarizada y a la gammagrafía ósea para detectar metástasis. La tomografia por emisión de positrones fue evaluada por Sanz G et al. en $1999^{23}$. Esos autores observaron en la tomografia por emisión de positrones más eficacia para mostrar recidiva local que la tomografía axial computarizada y menos eficacia que la gammagrafia ósea para detectar recidivas a distancia.

\section{Biopsia del lecho prostático.}

La biopsia ecodirigida del lecho prostático y de la anastomosis de la unión vesicouretral es un procedimiento diagnóstico de la recidiva local del cáncer de próstata después de la prostatectomía radical. Sin embargo, bajos volúmenes de la enfermedad que pueden ser detectados con niveles de PSA muy bajos pueden pasar desapercibidos y no ser diagnosticados con una biopsia del lecho prostático. Una biopsia positiva no excluye enfermedad sistémica ni una biopsia negativa excluye enfermedad local ${ }^{4}$. Según los análisis de Connolly JA et al. de $1996^{24}$ y Saleem MD et al. de $1998^{25}$ solamente un $25 \%$ de los pacientes diagnosticados de recidiva local con niveles de PSA inferiores a $1 \mathrm{ng} / \mathrm{ml}$ presentaban biopsia positiva del lecho prostático. En ese sentido, Lange $\mathrm{PH}$ et al. en $1990^{26}$ informaron de los resultados de la radioterapia local de rescate en 29 pacientes con fracaso de PSA después de la prostatectomía radical, 18 pacientes con biopsia de la unión vesicouretral positiva y 10 pacientes con la biopsia de la unión vesicouretral negativa. En el grupo de pacientes con biopsia positiva respondieron a la radioterapia 16 (89\%) de los pacientes y en el grupo con biopsias negativas respondieron 7 (70\%) de los pacientes, sin diferencias significativas entre los dos grupos.

Los pacientes con una cinética de PSA favorable, en la linea de los parámetros que hemos seleccionado en nuestro estudio, son buenos candidatos para recibir con posibilidades de éxito radioterapia en la celda prostática ${ }^{27}$. La dosis de radiación recomendada por la Sociedad Americana de Radioterapia y Oncología es de 64 Gy o ligeramente superior, administradas en fracciones de 1,8 a $2 \mathrm{~Gy}^{28}$. Los resultados libres de fracaso bioquímico de la radioterapia de rescate son muy variables, dependiendo de múltiples factores, y pueden estar según las series analizadas por Nelson et al. en $2003^{4}$ entre el 0 y el $55 \%$, con seguimientos que oscilan entre 3 y 10 años. En nuestro estudio los resultados libres de fracaso bioquímico con radioterapia de rescate en pacientes muy seleccionados fueron del $77,8 \%$ con un seguimiento medio de 2 años.

\section{CONCLUSIONES}

La radioterapia de rescate del lecho prostático es eficaz en casos muy seleccionados de pacientes con recidiva bioquímica después de la prostatectomía radical. El análisis de la cinética del PSA o comportamiento del PSA postoperatorio es un buen método para distinguir entre recidiva local y recidiva sistémica e indicar tratamiento con radioterapia en el lecho prostático, con intención curativa.

\section{REFERENCIAS}

1. Pound CR, Partin AW, Epstein JI, Walsh PC. Prostate-specific antigen after anatomic radical retropubic prostatectomy. Patterns of recurrence and cancer control. Urol Clin North Am 1997;24(2):395-406.

2. Loberg RD, Fielhauer JR, Pienta BA, Dresden S, Christmas P, Kalikin LM, Olson KB, Pienta KJ. Prostate-specific antigen doubling time and survival in patients with advanced metastatic prostate cancer. Urology 2003;62(6 Suppl 2):128-133.

3. Chatta G. Multiplex biomarker approach for determining risk of prostate-specific antigen-defined recurrence of prostate cancer. Rhodes DR, Sanda MG, Otte AP, Chinnaiyan AM, Rubin MA, Department of Pathology, University of Michigan School of Medicine, Ann Arbor, MI. J Natl Cancer Inst 2003;95:661-668. Urol Oncol. 2003;21(6):488. 
4. Nelson JB, Lepor H. Prostate cancer: radical prostatectomy. Urol Clin North Am 2003;30(4):703-723.

5. Ward JF, Blute ML, Slezak J, Bergstralh EJ, Zincke H. The long-term clinical impact of biochemical recurrence of prostate cancer 5 or more years after radical prostatectomy. J Urol. 2003;170(5):1872-1876.

6. Scheepe JR, Vis AN, Mickisch GH. Recurrence after radical prostatectomy for organ-confined prostate cancer. Onkologie 2003;26(4):330-333.

7. Moul JW. Variables in predicting survival based on treating "PSA-only" relapse. Urol Oncol 2003;21(4):292-304.

8. Cannon GM, Walsh PC, Partin AW, Pound CR. Prostatespecific antigen doubling time in the identification of patients at risk for progression after treatment and biochemical recurrence for prostate cancer. Urology 2003; 62(6 Suppl 2):2-8.

9. Partin AW, Pearson JD, Landis PK, Carter HB, Pound CR, Clemens JQ, Epstein JI, Walsh PC. Evaluation of serum prostate-specific antigen velocity after radical prostatectomy to distinguish local recurrence from distant metastases. Urology 1994;43(5):649-659.

10, Patel A, Dorey F, Franklin J, De Kernion JB. Recurrence patterns after radical retropubic prostatectomy: clinical usefulness of prostate specific antigen doubling times and log slope prostate specific antigen. J Urol 1997;158(4): 1441-1445.

11. Wu JJ, King SC, Montana GS, McKinstry CA, Anscher MS. The efficacy of postprostatectomy radiotherapy in patients with an isolated elevation of serum prostate-specific antigen. Int J Radiat Oncol Biol Phys 1995;32(2):317-323.

12. Sobin LH, Wittekind CH. TNM Clasificación de los tumores malignos. Barcelona. Pulso Ediciones, 2002;26-29.

13. Pisansky TM, Kozelsky TF, Myers RP, Hillman DW, Blute ML, Buskirk SJ, Cheville JC, Ferrigni RG, Schild SE. Radiotherapy for isolated serum prostate specific antigen elevation after prostatectomy for prostate cancer. J Urol 2000; 163(3):845-850,

14. Haab F, Meulemans A, Boccon-Gibod L, Dauge MC, Delmas V, Boccon-Gibod L. Clearance of serum PSA after open surgery for benign prostatic hypertrophy, radical cystectomy, and radical prostatectomy. Prostate. 1995;26(6):334-338.

15. Brawer MK, Kirby R. Antígeno prostático específico. Barcelona. Ediciones Médicas SL, 2001; 7-22.

16. Pound CR, Partin AW, Eisenberger MA, Chan DW, Pearson JD, Walsh PC. Natural history of progression after PSA elevation following radical prostatectomy. JAMA 1999; 281(17): 1591-1597.

17. Roberts SG, Blute ML, Bergstralh EJ, Slezak JM, Zincke H. PSA doubling time as a predictor of clinical progression after biochemical failure following radical prostatectomy for prostate cancer. Mayo Clin Proc 2001 jun;76(6):576-581.

18. Cher ML, Bianco FJ Jr, Lam JS, Davis LP, Grignon DJ, Sakr WA, Banerjee M, Pontes JE, Wood DP Jr. Limited role of radionuclide bone scintigraphy in patients with prostate specific antigen elevations after radical prostatectomy. J Urol 1998;160(4):1387-1391.
19. Older RA, Lippert MC, Gay SB, Omary RA, Hillman BJ. Computed tomography appearance of the prostatic fossa following radical prostatectomy. Acad Radiol. 1995;2(6): 470-474.

20, Kramer S, Gorich J, Gottfried HW, Riska P, Aschoff AJ, Rilinger N, Brambs HJ, Sokiranski R. Sensitivity of computed tomography in detecting local recurrence of prostatic carcinoma following radical prostatectomy. Br J Radiol 1997;70(838):995-999.

21. Kahn D, Williams RD, Manyak MJ, Haseman MK, Seldin DW, Libertino JA, Maguire RT. 111Indium-capromab pendetide in the evaluation of patients with residual or recurrent prostate cancer after radical prostatectomy. The ProstaScint Study Group. J Urol 1998;159(6):2041-2046.

22. Raj GV, Partin AW, Polascik TJ. Clinical utility of indium 111-capromab pendetide immunoscintigraphy in the detection of early, recurrent prostate carcinoma after radical prostatectomy. Cancer 2002 feb; 94(4):987-996.

23. Sanz G, Robles JE, Giménez M, Arocena J, Sánchez D, Rodríguez-Rubio F, Rosell D, Richter JA, Berián JM. Positron emission tomography with 18fluorine-labelled deoxyglucose: utility in localized and advanced prostate cancer. BJU Int 1999;84(9):1028-1031.

24. Connolly JA, Shinohara K, Presti JC Jr, Carroll PR. Local recurrence after radical prostatectomy: characteristics in size, location, and relationship to prostate-specific antigen and surgical margins. Urology 1996;47(2):225-231.

25. Saleem MD, Sanders H, Abu El Naser M, El-Galley R. Factors predicting cancer detection in biopsy of the prostatic fossa after radical prostatectomy. Urology 1998;51(2): 283-286.

26. Lange PH, Lightner DJ, Medini E, Reddy PK, Vessella RL. The effect of radiation therapy after radical prostatectomy in patients with elevated prostate specific antigen levels. J Urol 1990;144(4):927-932.

27. Nudell DM, Grossfeld GD, Weinberg VK, Roach M 3rd, Carroll PR. Radiotherapy after radical prostatectomy: treatment outcomes and failure patterns. Urology 1999;54(6): 1049-1057.

28. Cox JD, Gallagher MJ, Hammond EH, Kaplan RS, Schellhammer PF. Consensus statements on radiation therapy of prostate cancer: guidelines for prostate re-biopsy after radiation and for radiation therapy with rising prostate-specific antigen levels after radical prostatectomy. American Society for Therapeutic Radiology and Oncology Consensus Panel. J Clin Oncol 1999;17(4):1155.

Dr. A. Ojea Calvo

Hospital Universitario Xeral-Cíes de Vigo

Pizarro, 22

36204 Vigo (Pontevedra)

(Trabajo recibido el 18 marzo de 2004) 